\title{
Characteristics of Hospital Architectural Space
}

\author{
Na Wang ${ }^{1, a}$ and corresponding author: Jinguo Wang ${ }^{2, b, *}$ \\ ${ }^{1}$ Department of Anesthesiology, The First Hospital of Jilin University, Changchun, China \\ ${ }^{2}$ Department of Urology, The First Hospital of Jilin University, Changchun, China \\ awangna080613@163.com, bwangjinguolily@163.com
}

\begin{abstract}
Keywords: Hospital, Architecture, Space, Design.
Abstract. Architectural space of hospital contains two types of basic space, using space and contacting space. Using space bears various functional requirements. In hospital buildings, functional space including waiting, seeing a doctor, examining, treating, charging and taking medicine, belongs to using space. This kind of space must follow the functional requirements. It shall be carried out in accordance with the standard and primary design. Contacting space meets the demand of traffic and public activities, but it also has special function of avoiding infection in hospital.
\end{abstract}

\section{Introduction}

Chinese medical architecture is becoming more and more perfect. Many hospitals are under construction. On one hand, it will be a good thing and proves that people's health is becoming more and more big important. The construction of the medical building is not so simple, because many hospitals is an engineering of buildings, so a lot of functions will unify together. The rationality of the inside order must be ensured, but in the actual design process there will be many problems. Therefore, special attention must be paid to design problems. [1]

In hospital building, waiting hall, elevator hall, waiting room, waiting area, walkways, business consulting space and bathroom space belong to contact space. Contact space will use space together, the different spaces jointly meet the demand of patients go to a doctor. Within the hospital, streamline design is particularly important to make people's activities orderly and smoothly. It is the basic principle of streamline design which includes the shortest distance, least time of turning back and the least guidance. In essence, the hospital buildings are the traffic types, because it is one of the key points of the research of stream of people and regular activities.

\section{Interface of Architectural Space}

Definition. Interface is the transition of two kinds of medium, from one medium to another medium critical level. For buildings, space as a medium, which can be connected to the functional space and contact space is interface. Buildings are in space form, so the interface space can also be referred to pawn construction. It reduces the size of connection communication obstacle. It is the key to design interface in the architectural space. Interface between buildings is help to use function of architectural space. A relationship between the space and people is that people in the use of main body function of building (e.g., medical function), in order to meet the use requirements, the demand of people physiology and psychology, promote mutual exchanges the planning and design of architectural space. [2]

Architectural space has environmental quality of freedom, and a simple interface communication, comfortable, smooth and efficient. Architectural space interface is characterized by: enough space, sunshine, appropriate greening, touching the color. Its role is mainly to contact, buffer, think, recognition, consulting, rest, coordination and integration of function space. There is a change of the interface design, from the development environment of understanding to reshape the necessity and urgency of the hospital culture. [3]

Function of Interface. Most large public hospitals in China for decades or even hundreds of years of history, both form in the process of long-term development of a more profound cultural accumulation, 
as the political and economic system reform deepening and hospital's own development change. It needs hospital spirit, school training, core values and the core content of hospital culture, which refine its necessity. [4]

First, the hospital sustainable development can't leave the society from all aspects of life widespread recognition and support. In view of the present widespread for medical misunderstanding, by reshaping, it declares and practices the core culture, which can realize dual effects. [5]

Second, the return of the hospital public welfare needs cultural support. This transformation, in urgent need of the new hospital culture leads the cadres and workers to change idea as soon as possible, to get the direction, solidarity and to unify thoughts to improve services, improve the quality, cost control, convenience and benefit of the medical track. [6]

\section{Space Settings}

Personal Space Settings. Patients due to illness diagnosis and subsequent treatment in hospital, the most need is proper personal adjustment space, from too much interference, to maintain personal privacy, on the basis of this point is particularly important for patients in hospital at present, due to excessive hospital resources nervous and patients, hospital patients personal space Settings there can be room for improvement, the development trend of the current hospital design is for single room number of the extension and escort empty asked, in the process of ascension in hospitalized patients with physical and mental relaxation. [7]

In addition to the number of single room to expand, in bed room arrangement design, also should adopt the principle of personal space, the basis of ensuring patients for privacy requirements. The open end of the ward is an outdoor green plant environment, ward oriented side as a whole is relatively closed, only need a credit card or other access facilities. To enter, less set observation window on the door and other facilities. [8]

Public Space Setting. In addition to patients with illicit close space, hospital public space design is a crucial and should be actively using a variety of patterns to thoughts of information transmission, the transmission requires public space, often in the traditional design of hospital for public space of the point defect not neat, er, not to carry on communication between the personnel and information, in practice, however, this can lead to the patient and family is in a state of anxiety, bright and open public space. [9] Help patients achieve physical and mental relaxation, easy to blend step and the medical staff to communicate, to ensure the patient's psychological and physiological active reply, guarantee the existence of its social security. [10]

Therefore, the appropriate placement of the ward is essential for the design of public space, at the same time choose bright color space decorate, for patients and their families to provide newspaper, Internet and other modern basic facilities, the psychological distance between closer to patients and hospital staff, to provide a more humane treatment experience. [11]

\section{The Design Proposal of Modern Hospital Building}

The Design of Humanized Concept. The basic problem in the design of hospital buildings is clear. The main body of service includes both patients and medical staff. The design should meet their physiological and psychological needs, material and spiritual needs. The needs of the people, the feelings of people, and human emotion is the first element to consider when building is designed. [12] Correctly classified by server (patients) and server (staff) space, create a quiet and tastefully laid out, halcyon, rich the milk of human kindness, medical environment, meet the needs of patients, medical staff, and other people.

Health Environment. In hospital cross infection is a serious problem: the patients of bacteria or virus is carried, between patients treated with direct contact (indirectly), or contact the hospital to dust in the air, medical sewage or cause infection chance again, so you must in aspects of architectural design, reduce the occurrence of cross infection. First of all, the layout of hospital makes every effort to avoid 
cross of patients in various departments, take all organization form layout, more strictly to avoid route across each other, reduce the contact between the patients. [13]

\section{Conclusions}

The construction of modern hospital environment is not only to create a good working environment for staff, but also to create a good medical environment for patients. To get out of the past error which is only according to doctors, nurses, and logistics department staff need to design, a benign environment should be built for doctors and patients. The center of the core purpose is to service patients.

Outstanding design concept of hospital landscape makes full use of the courtyard space, taking point line field. It is a combination of plane and solid, and as much as possible to increase the total greening, and USES the representative of green life as the mass-tone attune of the hospital environment, setting up the role of furniture to improve the construction and road surface hardening of curt feeling. It makes it a pleasant place for rest and rehabilitation and easy to patients.

\section{References}

[1] Yiska Goldfeld, Johann Arbocz, Alan Rothwell. Design and optimization of laminated conical shells for buckling[J]. Thin-Walled Structures. 2004 (1)

[2] Kumarapollai K.N., Coull A. Journal of the Structural Division. American Society of Continuing Education. 1972

[3] Swaddiwudhipong S., Balendra T., Quek S.T., Lee S.L. Computer Program For The Analysis Of Asymmetric Frame-Shear Wall Structures. Computers and Structures . 1986

[4] Martin Hernandez-Marin, Thomas J. Burbey. Fault-controlled deformation and stress from pumping-induced groundwater flow[J] . Journal of Hydrology. 2012

[5] K. A. Zalka. Torsional analysis of multi-storey building structures under horizontal load[J]. Struct. Design Tall Spec. Build. . 2010 (2)

[6] Boudaud C, Baroth J, Daudeville L. Influence of joint strength variability in timber-frame structures:propagation of uncertainty through shear wall finite element models under seismic loading. Canadian Journal of Civil Engineering. 2016

[7] Jinhan Kwon, Wassim M. Ghannoum. Assessment of international standard provisions on stiffness of reinforced concrete moment frame and shear wall buildings. Engineering Structures. 2016

[8] Vuddandam R B, Toutanji H. Approximate solutions to coupled shear walls on fixed and flexible foundations. Modern Applied Science. 2013

[9] Schmit LA. Structure design by synthesis. Proceedings of the 2nd Conference on Electronic Computation . 2011

[10] Rosenblueth E, Elorduy J, Rosenblueth E. Response of linear systems to certain transient disturbances. 1969

[11] Kaushik S, Dasgupta K. Seismic damage in shear wall-slab junction in rc buildings. Procedia Engineering. 2016

[12] Tso WK, Biswas JK. General analysis of nonplanar coupled shear walls. Journal of the Structural Division of the ASCE. 1973

[13] Surana M, Singh Y, Lang D H. Seismic performance of mid-rise rc frame and frame-shear wall buildings designed for Indian codes. Journal of Structural Engineering. 2016 